She returned to London, where she was employed at secretarial work, and by my advice put herself under the care of a tutor for defective speech. It will easily be understood that the faulty voice production of a lifetime was not to be overcome after a lesson or two, but she was diligent and intelligent, and she gradually acquired complete control so as to be able to talk quite correctly, if at the first a little pedantically. Her greatest obstacle was in dispensing with the glottal stop of which such people make so much use as a substitute for labials and dentals.

\section{RIGHT BRANCH BUNDLE BLOCK.}

W. S. MALCOLM, C.M., M.D.,

ELECTROCARDIOGRAPHER AND ASSISTANT PHYSICIAN, DUNDEE ROYAT INFIRMARY.

Electrocardiography is the only means of discovering the existence of disorders of heart conductivity in the terminal branches of the bundle of His. Right branch bundle block does not imply a disturbance of rate or of regularity, and, being an expression of myocardial degeneration, recognition is of importance for prognosis. It may be that visible evidence of abnormal activity of the left ventricle is present and that a reduplicated first sound is heard, but these signs are not pathognomonic of the condition.

A normal electrocardiogram is shown in Fig. 1: p represents auricular activity and QRST ventricular; QRS is called initial ventricular, for it is recorded as the ventricles are passing into activity, and $\mathbf{T}$ is terminal ventricular. The ventricular curve arises from the change of potential induced as the wave of contraction passes through the ventricles, and it is really the algebraic sum of the deflecticns which would be produced by the right and left ventricles contracting separately. Lewis ${ }^{1}$ has shown that $\mathbf{R}$ of Lead 1 and s of Lead 3 are mainly left ventricular events. These preliminaries help the understanding of what takes place when the contraction stimulus fails to pass along the right branch in right branch bundle block.

Having traversed the bundle of $\mathrm{His}$, this stimulus finds in right branch block the right branch impermeable, or that conduction is slower than it is along the left branch. Consequently the contraction stimulus reaches first the left

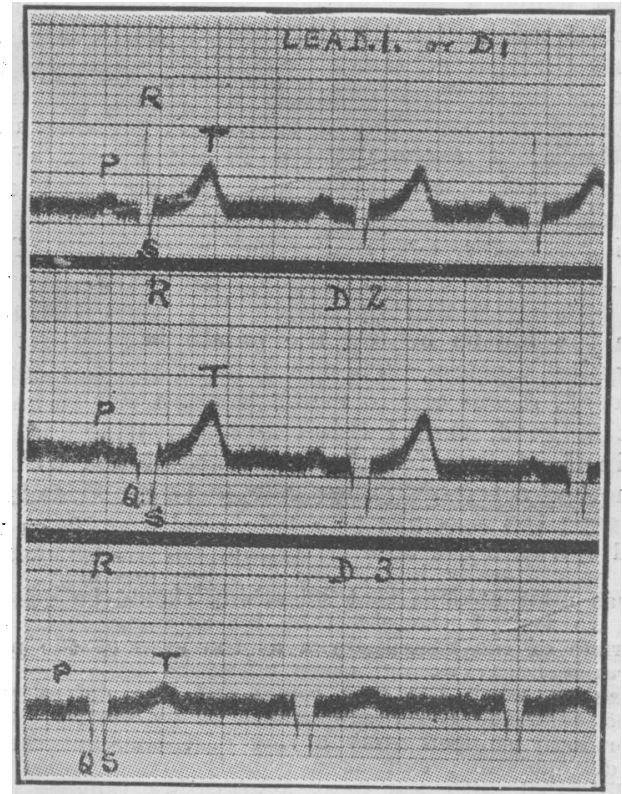

FIC. 1.

ventricle, and if the right branch is impermeable the stimulus passes from left to right ventricle through the medium of myocardial fibres common to both ventricles. Thus the right ventricle is later than the left in passing into contraction. Now QRS represents the initial activity of both ventricles; consequently the duration of the initial ventricular contraction is prolonged. The lengthening of the QRs period is the principal feature of branch block. Normally it does not exceed 0.10 second, and in health its duration is less than one-third of the total duration of ventricular activity (QRST). In branch block it exceeds

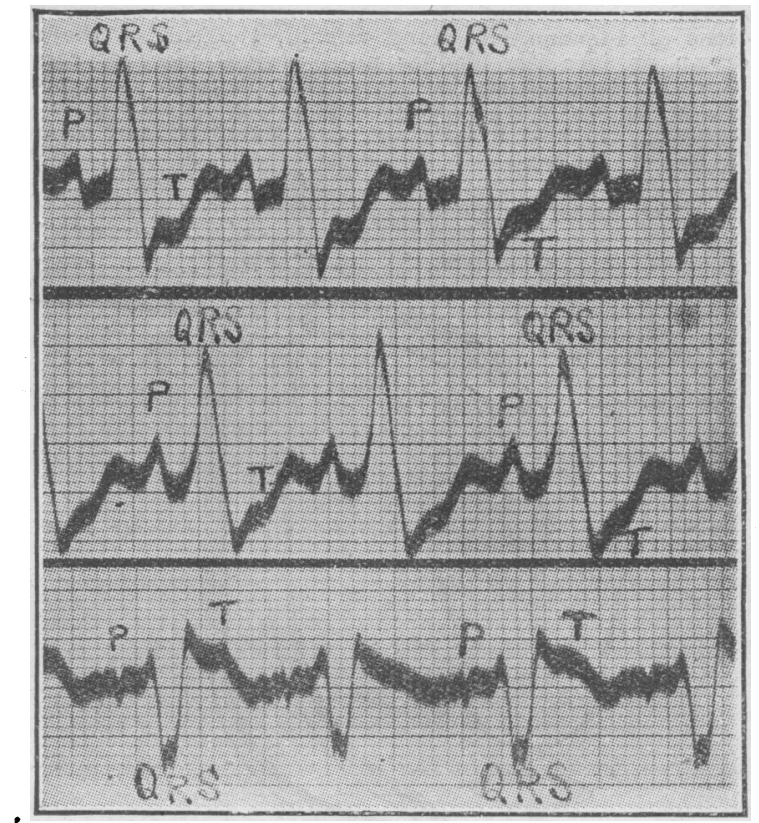

FIG. 2.

0.10 second and it lasts longer than one-third of -QRST. The fact that the contraction concerns first the left ventricle and then passes to the right explains the beading or notching frequently seen on the group deflection QRs.

The second characteristic of branch block is that the terminal wave $\mathrm{x}$ of the ventricular deflection has a direction opposite to that of the initial one (QRS). If QRS is upward, $T$ is downward, and if QRs is downward, $T$ is upward. Having decided from these two signs that there is a block of one branch of the bifurcation of the bundle of His, we have to determine whether it is left or right. If the main spike of the QRS group is upward in Lead 1

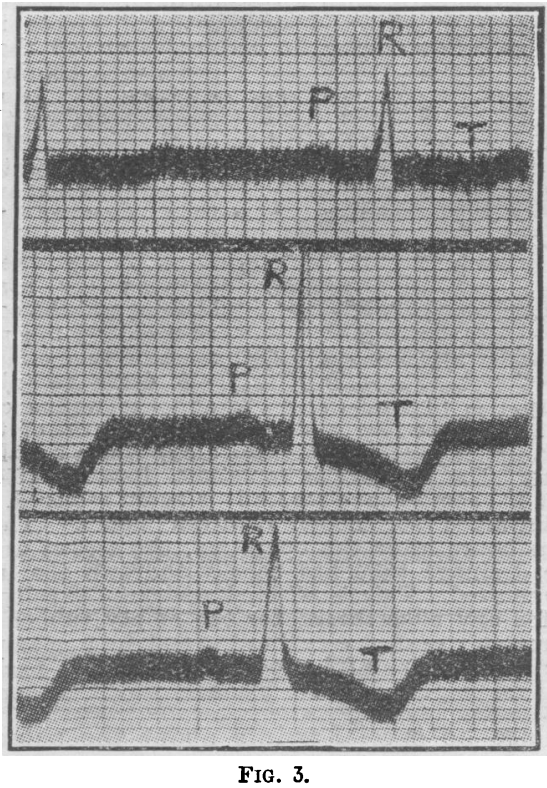

and downward in Lead 3, there is a condition of predominance of the left side, for $\mathrm{R} 1$ and s3 are mainly left ventricular events. ${ }^{1}$ This implies that the left branch is intact and that the right fails in conductivity, the condition of right branch bundle block.

The condition in a spinster, aged 60 , with signs of heart failure with oedema and congestion, clubbed finger-tips, 
triple rhythm, daily range of temperature $97^{\circ}$ to $98.40^{\circ}$, is illustrated in Fig. 2. The diphasic ventricular complex is shown with characteristics already noted. The initial phase of ventricular activity is necessarily prolonged, for the stimulus has first to traverse the left ventricle before 'reaching the right.

A record closing five months' observation is shown in Fig. 3. The tracing shows reversion to the physiological type, but the steep negativity of $\mathrm{T} 2$ and $\mathrm{r} 3$ is ominous.

Of three cases encountered two were males. All had reached the degenerate period of life, with thickened arteries and high systolic and diastolic readings, and with symptoms indicative of cardiac insufficiency. The associated cardiac lesions were respectively mitral, aortic (Wassermann reaction positive), and hypertrophy (with renal cirrhosis), the common factor being myocardial with degenerative arterial change.

Branch block adds to the gravity of prognosis, as the much quoted statistics of Willius ${ }^{5}$ show. In an analysis of twenty-four cases of branch block, Cowan and Bramwell ${ }^{4}$ found that in only one case did the block show a tendency to disappear. Lewis, ${ }^{6}$ Ritchie,${ }^{7}$ and Hewlett ${ }^{8}$ have recorded cases in which block was present as a temporary manifestation in association with febrile or toxic disturbance, as in the case here quoted. In Hall's case ${ }^{9}$ the block was permanent but stationary, in contrast with the ordinary rule that the lesion, if chronic, is progressive.

I wish to acknowledge my indebtedness to Dr. Foggie, in whose ward the patient was.

\section{REFERENCES}

1 Clinical Electrocardiography, p. 28.2 Gross: The Blood Supply of the Heart (quoted in 4). 3 MacIlwaine and Campbell: British Mrdicil JoliRNAL, 1923, ii, 456. 4 Quart. Journ. Med., No. 73, October, 1925 5 Willius: Clinical Electrocardiograpliy. ${ }^{6}$ BRITISH MEDICAL JOLRNAL, $1913,484.7$ Cowan and Ritchie : Diseases of Heart ${ }_{8}^{8}$ Heart, $1921,9,1$ 1913, 1, 484.

\section{TUBERCULOSIS AND MILK.*}

NATHAN RAW, C.M.G., M.D., LORD CHANCELLOR'S VISITOR IN LUNACY.

Is the British Medical Joursal of 1903 I ventured to suggest that bovine bacilli conveyed by milk played an important part in causing tuberculosis in children. This has since been confirmed by many other workers, and is How accepted as a serious menace to child life.

The International Congress on Tuberculosis which was held in Washington in 1908 will always be remembered on account of the discussion which took place on the part played by the bovine bacillus in the production of tuberculosis in man. We were honoured on that occasion by the presence of the great master, Robert Koch; and those of us who took part in the secret session will never forget it. Up to that time Koch had not publicly admitted that bovine infections in man were possible, but he later modified his views, and we are all now agreed that an important amount of tuberculosis, especially in children, is directly conveyed from the dairy cow.

At that conference $I$ read a paper, entirely based on clinical and hospital observation, pointing out the possibility that surgical tuberculosis in children, such as onlarged lymph glands, mesenteric disease, and affections of bones and joints, were separate and distinct infections from those of pulmonary tuberculosis, and might be caused by milk. The brilliant pioneer work of our president, Thebold Smith, on the role of the bovine bacillus, stimulated many workers, myself included, to further investigation, and it is particularly fitting that, eighteen years afterwards in this city of Washington, Dr. Park should tell us of the immense reductions in infant mortality and suffering as the result of their researches.

The incidence of bovine tuberculosis varies in different countries, and depends upon the extent to which cattle are infected with tuberculosis and the amount of uncooked milk, butter, cheese, and other dairy products consumed. In England and Scotland dairy cows are very heavily

* Read before the International Conference on Tuberculosis, Washington, October 2nd, 1925 . affected, with the result that surgical tuberculosis is very common in children.

In the light of our present knowledge we cannot say with certainty which particular strain of bacillus has caused the infection. We are accustomed to recognize three types of tubercle bacilli: human, bovine, and avian. I feel convinced that there are several intermediate strains of bacilli, which we cannot yet recognize with certainty, and it is not possible to say without bacteriological and animal tests to which group those from any lesion belong. For this reason, for the purpose of determining milk infections, clinical and hospital evidence is valuable.

Pulmonary tuberculosis is in a class by itself, and is practically always caused by the human type of bacillus, which also causes laryngitis, secondary intestinal ulceration, and fistula. It is rare for such cases to develop any other lesions. On the other hand, bovine infections in human beings rarely cause pulmonary lesions; in fact, they seem to be antagonistic to each other, and it is possible that a primary bovine infection, such as enlarged neck glands, may produce immunity against pulmonary tuberculosis foi life.

Should this theory prove correct, the beneficial effects of sterilization of tuberculous milk may be followed by diminished resistance to human infection in the form of pulmonary tuberculosis. In any case it is imperative that the milk supply be pure and safe for children.

Avian bacilli, though considered to be non-pathogenic to man, may contaminate a milk supply, but with what effect we do not know. In Denmark the danger of infection of pigs by avian bacilli is so real that legislation has been introduced forbidding chickens on the farm.

To sum up. In my opinion-

\section{Human bacilli produce:}

1. Pulmonary tuberculosis.

2. Tuberculous laryngitis.

3. Secondary intestinal ulceration.

4. Fistula in ano.

Bovine bacilli produce :

1. Enlarged lymph glands.

2. Abdominal tuberculosis.

3. Lesions of bones and joints.

4. Meningitis.

5. Acute miliary tuberculosis.

6. Lupus.

7. Rarely, secondary extension to lungs.

These infections are antagonistic to each other, and human and bovine bacilli are rarely found in the body at the same time. The practical measures that should be taken against bovine infection are cradication of tuberculosis from dairy cows and pasteurization of all milk for human consumption.

Eradication will for a long time be impossible; pasteurization is a safe and certain remedy. The results in several large cities where compulsory pasteurization is law are truly wonderful, and have resulted in the saving of the lives of thousands of children every year, and endless pain and suffering with lifelong deformity. I sincerely trust the splendid pioneer work in New York and other American cities will soon be universal. At the same time it is imperative that every effort should be made to stamp out tuberculosis in dairy cattle.

It is estimated that in England about a million dairy cows are affected with tuberculosis. The cows with udder disease are, of course; the most dangerous. The compulsory seizure, isolation, and slaughter of these cows is a slow, laborious, and very costly procedure. The new Tuberculosis Order recently made in England will in time have a most beneficial effect in removing diseased cows from dairy herds, and so reducing the amount of bovine infection in children. In addition it is to be remembered that the financial loss to stock breeders and farmers from tuberculosis is enormous.

The question of the possible immunization or vaccination of cattle against tuberculous infection is of supreme importance, and as practically all cattle are born free of any tubercle-infection taking place after birth-the problem ought to admit of a solution.

I would like to lay before this conference the results of my own work, during the last eighteen years, in attempting to produce immunity in animals against tuberculosis. In 1906 Professor Koch gave me in Berlin a pure culture on 